\title{
Reconstrucción de una armadura de cubierta de lazo, en la iglesia parroquial de Almenara de Tormes (Salamanca)
}

\author{
Reconstruction of a interlacing pattern of a wood ceiling in the \\ church of Almenara de Tormes (Salamanca)
}

M. C. Fernández-Cabo

\section{RESUMEN}

El artículo describe el proceso de restauración de la armadura de cubierta de la nave principal de la iglesia románica de Almenara de Tormes, Salamanca (España). El techo de la armadura de par y nudillo del siglo XVI estaba oculto por un entablado, que al ser retirado permitió apreciar unas entalladuras que evidenciaban la existencia de una lacería que se había perdido. En base a la regularidad geométrica de esos cortes o trazas aparecidos en los nudillos, fue posible realizar una reconstrucción completa de la lacería. Se investigó sobre los trazados de lazo que pudieran encajar en esos cortes, rebajes o entalladuras, analizando otros trazados de lazo de la época y lugar histórico. Después de comprobar las medidas y ángulos precisos de las trazas de los nudillos se formularon algunas hipótesis de trazado de lazo y se consiguió desarrollar un trazado que encajaba a la perfección con los restos existentes. Como resultado de esta investigación se pudo proceder a la elaboración de un proyecto de restauración procediendo a continuación a la reconstrucción completa del artesonado hasta en sus más pequeños detalles.

\section{SUMMARY}

The paper describes the restoration process of a Romanesque church in Almenara de Tormes, Salamanca (Spain). The collar roof type timber frame, from XVI's, was hidden and showed cuts at the bottom face from a lost wooden ceiling. On basis of these cut collar beams, that original ceiling was able to be rebuilt, because these constructions have a regular geometrical design. A precise geometrical reconstruction of the existing timber structure was the first step. The geometry of the cuts allowed, without any dou$b t$, to know the original design. This is possible because these timber structures, from the XIV's and specific from Spain, are based on strict geometrical design. A restoration architectural project was developed based on that previous research work. This work explains the research work and describes both the architectural project and the construction works.
Palabras clave: estructuras de madera, artesonados, armaduras de cubierta, trazados de lazo, geometría decorativa.
Keywords: timber frame, wooden ceiling, roof ceiling, interlacing pattern, decorative geometry.

\footnotetext{
(*) ETS de Arquitectura de Madrid, Departamento de Construcción y Tecnología Arquitectónica, Universidad Politécnica de Madrid, (España) 
1. Vista general del estado original de la armadura con el almizate oculto por un entablado.

2. Sección general de la armadura de par y nudillo con un glosario de términos para denominar sus distintas partes o piezas.

\section{INTRODUCCIÓN: MEMORIA HISTÓRICA Y DESCRIPTIVA}

La armadura de cubierta -estructura de cubierta construida en madera- se sitúa sobre una iglesia salmantina de fundación románica sobre la que a lo largo de la historia se han practicado distintas intervenciones,
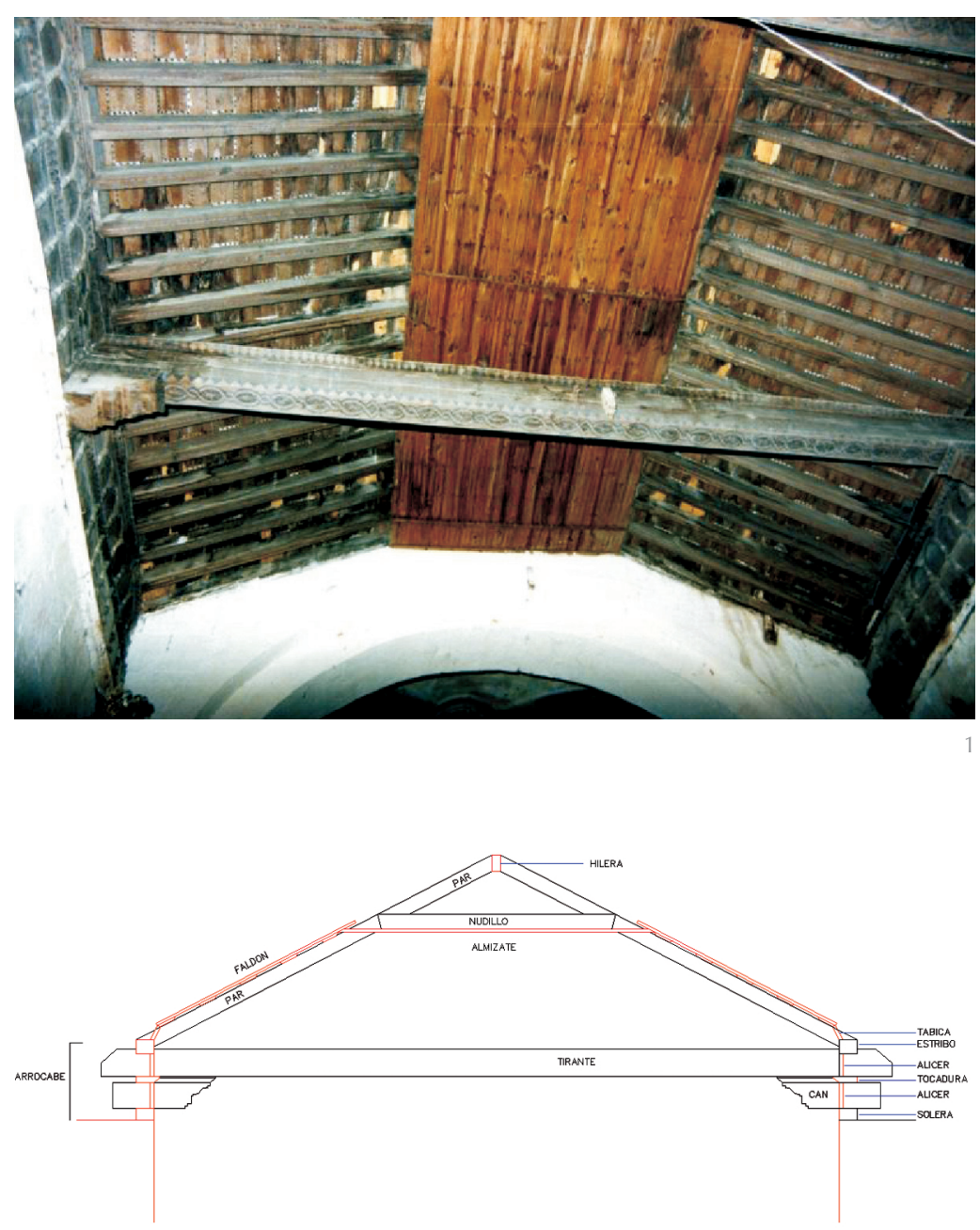

siendo la más importante la que se ejecutó entre los siglos XVI y XVII. A partir del análisis de los pergaminos originales existentes en el archivo diocesano y del análisis estilístico de sus portadas, los fundamentos de ésta iglesia se pueden fechar hacia el año 1164. Sin embargo la armadura de cubierta que nos ocupa, a juzgar por su tipología, trazas y decoración, debemos situarla entorno a finales del siglo XVI o principios del XVII, cuando posiblemente se procedió a sustituir la cubierta románica por la actual cubierta que ha llegado hasta nosotros.

Se trata de una armadura de par y nudillo con tirantes simples sobre canes moldurados; el par es cada una de las barras inclinadas y el nudillo es la pieza horizontal usada como contrarresto de los pares. Las gualderas o fal- dones se realizan al modo /lano. Esta solución también conocida como de cinta y saetino ha sido la manera más básica de resolver la cubierta de estas armaduras históricas. Los pares Ilevan decoración tallada en su eje central con una calle de pequeñas cuñas realizadas a golpe de gubia, además del clásico ranurado o agramilado practicado en los papos -cara de intradós-; los tirantes, se decoran con una tocadura en su cara superior al modo de una pequeña cornisa y llevan decoración policroma en los laterales y en la cara inferior consistente en un lazo desarrollado entre círculos alineados de distinto diámetro. El arrocabe o friso de arranque de la armadura, es de doble alicer-tablas de forro del friso- con decoración de talla de columnillas adosadas, dándole un carácter claramente renacentista en contraste con la decoración de lacería del almizate, cuyas trazas dejadas en los nudillos anunciaban claramente la pervivencia de tendencias estilísticas de influencia mudéjar, que curiosamente coexisten con las nuevas tendencias renacientes de la época (1).

El almizate o harneruelo de la armadura -techo o plano horizontal de una armadura de par y nudillo- se hallaba oculto bajo un simple entablado de madera (Figura 1), para evitar visualizar la ausencia de los alfardones entre los nudillos. Estos alfardones o tablas colocadas como forro de la armadura, estarían alojados entre unas ranuras o acanalados que se observan en sendos laterales de los nudillos (Figura 2). Supuestamente, los alfardones desaparecidos, así como los taujeles o tablillas de poco grueso que constituían el trazado de lazo, tuvieron que tener así mismo algún tipo de policromía, sobre todo en los zafates, similar a la decoración de punteaduras pintadas que se observaron como remate de cintas y saetinos en los faldones.

No se conocen las causas de la desaparición de éstas piezas pero en principio existen dos posibilidades. La primera es que el elevado grado de deterioro de estas piezas de pequeña sección haya motivado el retirarlas de la vista; conviene recordar en este sentido, que los ataques habituales a que se ve expuesta la madera -hongos y xilófagos-afecta en mayor medida a las escuadrías de pequeña sección, como es el caso de la tabla y los taujeles. La segunda posibilidad es que pudo existir un expolio de la decoración de lazo, cuyos taujeles y alfardones, al tratarse de pequeñas piezas de madera fáciles de extraer sin menoscabar de manera importante la resistencia de la estructura de cubierta, pudieran terminar en algún museo o servir para reconstruir otra lacería en otro edificio, caso que ocurría con cierta frecuencia (2) al tratarse de objetos que se mueven entre el mundo de lo inmobiliario y de lo mobiliario. Este es el caso de algunas lacerías procedentes de Valencia de Don 
Juan, León, y que se encuentran en el Museo Nacional de Artes Decorativas de Madrid, que curiosamente también es un trazado inspirado en ruedas de lazo de 8 y de 4 con algunos zafates harpados similares a los que nos encontramos en el trazado que estamos analizando.

A partir de estos restos pasaremos a explicar el procedimiento seguido para conseguir una restitución virtual del trazado de lazo ejecutado hacia el siglo XVI. El elevado grado de certidumbre de la hipótesis propuesta en la investigación del trazado, fue la justificación requerida para realizar una reconstrucción completa de la armadura de lazo, tal como se supone que fue ejecutada originalmente.

Este tipo de trabajos de restauración ya habían sido iniciados en España por Enrique Nuere, quién publica uno de sus primeros trabajos como apéndice a unos de su primeros libros (3). Se trataba de la recuperación de los restos de un almizate existente en el Museo Nacional de Arte Hispanomusulmán de la Alhambra, Granada, y después de este caso han seguido otros, realizados en algunas de sus numerosas intervenciones en armaduras de cubierta con lazo del patrimonio histórico español.

\section{EL ESTADO INICIAL}

Cuando se redactó el proyecto inicial de restauración de las obras - del que mostramos los planos de sección de la armadura (Figura 2)-, sólo se contaba con una apreciación visual externa del intradós de la armadura de cubierta, ya que por circunstancias de orden administrativo que no vienen a cuento en este artículo, hubo que redactar un primer proyecto sin contar con la información que posteriormente se obtuvo cuando dieron comienzo las obras. Se ha aprovechado esta sección para ilustrar gráficamente el léxico particular del mundo de las armaduras de cubierta tradicionales españolas.

Una vez iniciadas las obras de restauración se ordenó practicar una cata en la tarima que ocultaba el almizate. Esta cata puso al descubierto la presencia de nudillos con entalladuras por su cara de intradós, con lo que de inmediato se ordenó desmontar todo el entablado del almizate poniendo al descubierto todo el conjunto de nudillos tallados que evidenciaban los restos de lo que en su día constituyó una completa lacería en el almizate.

Además de las entalladuras o cortes practicados sobre el papo de los nudillos, se pudo apreciar una acanaladura longitudinal a ambos lados, donde habitualmente se alojaban los alfardones que supuestamente debieron ir insertados al modo de guillotina entre las acanaladuras (Figura 3). Era obvio pues que los taujeles o cintas del lazo desaparecidas, tendrías de grueso la misma profundidad de rebaje practicado en las entalladuras de los nudillos, a los cuales atravesaban enrasados por el intradós.

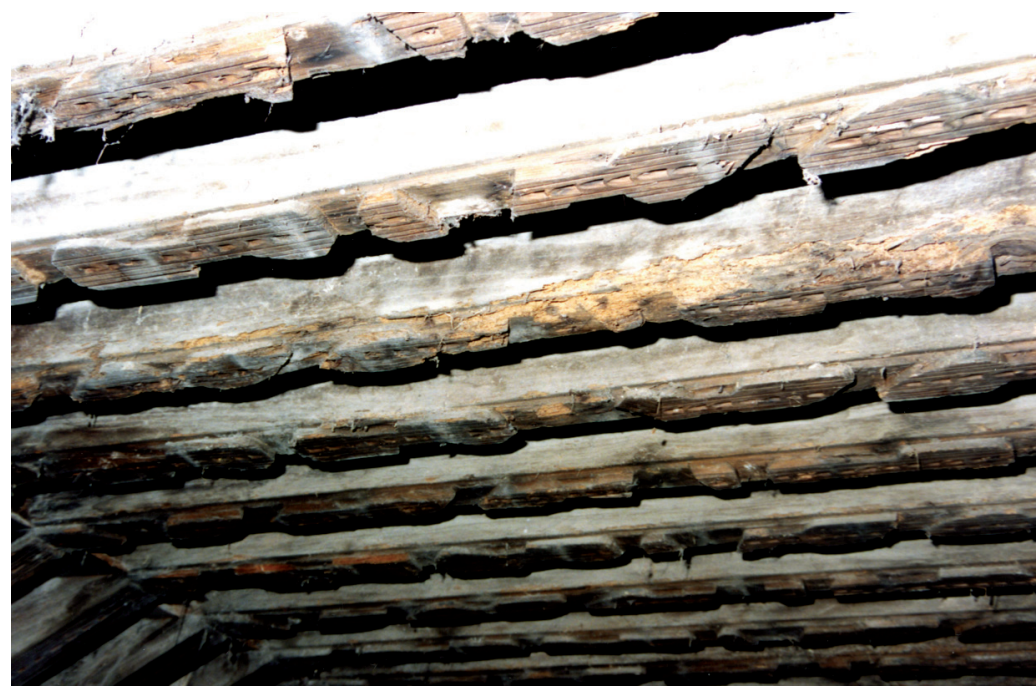

Esta lacería es un caso particular de mezcla entre los dos tipos constructivos básicos utilizados para la ejecución de lacerías en madera (4): lacería apeinazada -realizada a base de peinazos de igual sección que las piezas estructurales-y lacería ataujerada -realizada a base de taujeles-. Las cintas del lazo situadas sobre los nudillos, se tallan sobre estas piezas estructurales, mientras que el resto de cintas del trazado se ejecuta con pequeñas tablillas o taujeles, que se acoplan sobre las entalladuras de los nudillos y se clavan sobre la tabla de los alfardones.

\section{HIPÓTESIS DE RECONSTRUCCIÓN DEL TRAZADO DE LAZO}

Una vez puestos al descubierto los nudillos tallados y después de realizar las primeras actuaciones de limpieza y saneamiento, se pudo dar comienzo a los trabajos de toma de datos realizando una medición exacta de los cortes practicados en los nudillos, tanto en lo relativo a los ángulos de corte y distancias entre los mismos. Así mismo se tomaron medidas relativas a la relación de calle y cuerda -anchura del nudillo y separación entre ellos- ya que es fundamental en el análisis de cualquier trazado de lazo, así como las pauta o secuencia de repetición de las trazas.

Como resultado de este primer análisis de las trazas de corte, se pudieron reducir a dos los tipos de trazas diferenciadas que iban a permitir el desarrollo total de la lacería; también se comprobó que las trazas eran simétricas con respecto al eje longitudinal del almizate, y con relación al transversal del conjunto
3. Vista del detalle de los cortes pracicados en los nudillos para alojar la lacería desaparecida. 
4. Secuencia de cortes o trazas existentes entre los restos de los nudillos de la armadura que pudieron medirse y dibujarse con una cierta precisión y luego han servido para la recuperación de la lacería.

5. Primera hipótesis de cartabones a partir de la geometría de un octagrama. La confirmación de los cartabones, A, B, y C, ha sido plenamente congruente con las entalladuras practicadas en los nudillos existentes.

6. Generación de un lazo basado en una estrella de 8 puntas.

7. Estrella de 8 y desarrollo de una primera hipótesis de lazo que encajara entre los cortes de los nudillos. de las dos piezas. A continuación se pasó a confeccionar un primer plano de la secuencia completa de las trazas o entalladas existentes en el conjunto de todos los nudillos (Figura 4).

Este primer plano de trazas (Figura 4) constituyó el dato objetivo sobre el cuál se podrían establecer las primeras hipótesis de reconstrucción virtual del trazado completo del lazo, implementando los taujeles necesarios para ello. Los primeros pasos en este método de trabajo consistieron en analizar los ángulos de corte para saber a qué familia o familias de lazo podían corresponder, y que cartabones se había utilizado (5), siguiendo para ello el método de los cartabones descrito por López de Arenas (6), interpretado y sacado a la luz por Enrique Nuere (2) (3).

Algunos cortes contenían ángulos como los de $45^{\circ}$ o su mitad $22,5^{\circ}$, fácilmente identificables como pertenecientes a la familia de lazos de 4 y 8 , con lo que inmediatamente esta fue la
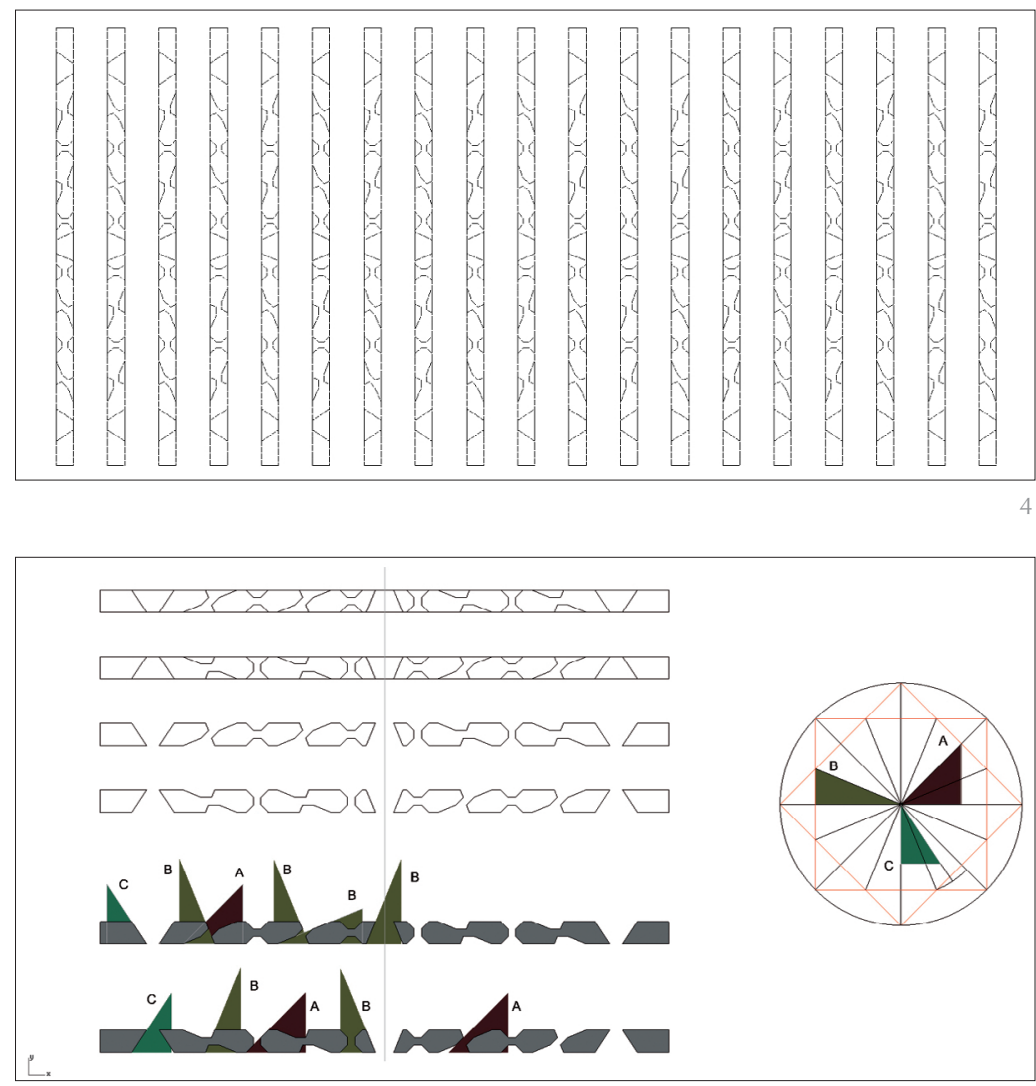

primera línea de trabajo a seguir. En base a esta primera hipótesis de lazo de 4-8, se dibujó una estrella de ocho puntas girando un cuadrado $45^{\circ}$ sobre su centro, dando como resultado una figura conocida como octograma. De este octagrama se sacaron los cartabones primarios, secundarios y terciarios, dando como resultado los siguientes cartabones (Figura 5):

- Cartabón A: cola $=$ cabeza $=\pi^{1 / 4}=45^{\circ}$ (también conocido como cuadrado)

- Cartabón B: cola $=\pi 1 / 8=22,5^{\circ}$ cabeza $=\pi 3 / 8=67,5^{\circ}$ (también conocido como cartabón de ocho)

- Cartabón C: cola $=\pi 3 / 16=33,75^{\circ}$ cabeza $=\pi 5 / 16=56,25^{\circ}$

(también conocido como blanquillo).

La cola del cartabón es el ángulo más agudo y la cabeza del cartabón es el ángulo complementario de la cola. En todos los cartabones uno de los ángulos es recto, ya que se construyen alojados en una cambija o medio círculo, y por el teorema del arco capaz, el ángulo que forman los catetos ha de ser recto $-90^{\circ}-$. Se construyen de esta manera para minimizar el número de éstas herramientas (7).

Estos cartabones se comprobaron sobre las trazas que se habían medido en los nudillos y se comprobó que no se precisaban más cartabones que éstos para realizar todos los cortes existentes. Con ello quedaba confirmado que la primera hipótesis de la familia de lazo buscada era correcta: se trataba de un trazado basado en los cartabones de la rueda de lazo de 8 , a la que le corresponde los citados cartabones de cuadrado, de ocho y el blanquillo.

Con esta confirmación ya se estaba en condiciones de buscar analogías visuales con otros trazados de lazo 4-8, conocidos en esa época y en esa región, para después pasar a formular unas primeras hipótesis de trazado que encajaran en las trazas existentes.

Los primeros dibujos se hicieron utilizando la relación calle/cuerda existente, y rotando esta crujía 8 veces en torno a un eje, formando una estrella similar a la que forman dos cuadrados girados $45^{\circ}$ (Figura 6). A partir de esta estrella de 8 situada en el eje longitudinal del almizate (Figura 7) que divide a la mitad a los nudillos, se comprobó que encajaba muy
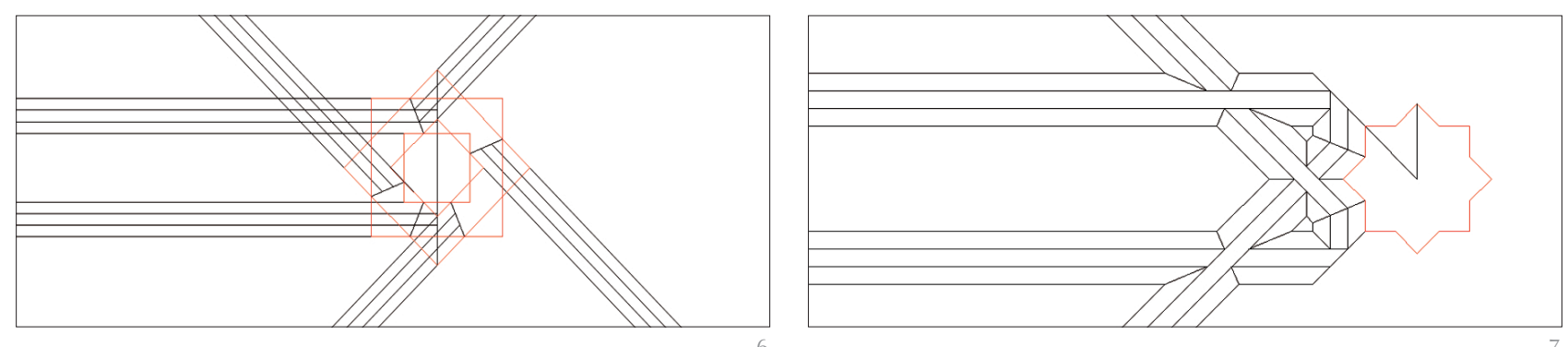
bien en los cortes existentes si se planteaba la hipótesis de que esta estrella se repitiera con alternancia de una calle si y otra no. De esta manera se fue colocando una estrella de ocho alternadamente (Figura 8) y a partir de ahí se intenta resolver el trazado en las calles que iban quedando entre las estrellas (Figura 9). En seguida se pudo comprobar que encajaba el clásico lazo castellano de 4, tan usual en las armaduras de esta época, entre los siglos XIV y XVII, y del cual podemos citar como referencia histórica al utilizado en el almizate de la armadura del Sancti Spiriti, de Toro en Zamora, fechada hacia finales del s. XIV.

Una vez establecida la relación entre los lazos de 8 y el lazo de 4, el paso siguiente consistió en desarrollar o desculatar-conectar otra rueda de lazo a través de las aspillas de la rueda inicial- las estrellas originales (Figura 10), inspirándose para ello en trazados de la época y de la zona geográfica del reino de León al que territorialmente pertenecía esta villa. Así fue fácil comprobar que se había utilizado en el desarrollo del argumento central una red de lazo conocida como lazo leonés (8) parecida a la que se encuentra en la armadura del Hostal de San Marcos de León, y en otras armaduras leonesas de la época (9). Este mismo diseño de trazado también ha sido utilizado con frecuencia para decorar el espacio entre tirantes pareados en varias armaduras situadas en Andalucía (10), dónde es más habitual el pareado de los tirantes.

Una vez comprobada esta hipótesis de trazado de lazo sobre los cortes de los nudillos existentes (Figura 11), sólo restaba solucionar el remate del lazo contra las gualderas o intradós de los faldones. En esta zona, las trazas próximas al quiebro del plano mostraron la solución de aspillas en cruz, con lo que el trazado ya quedaba resuelto en todas sus pautas.
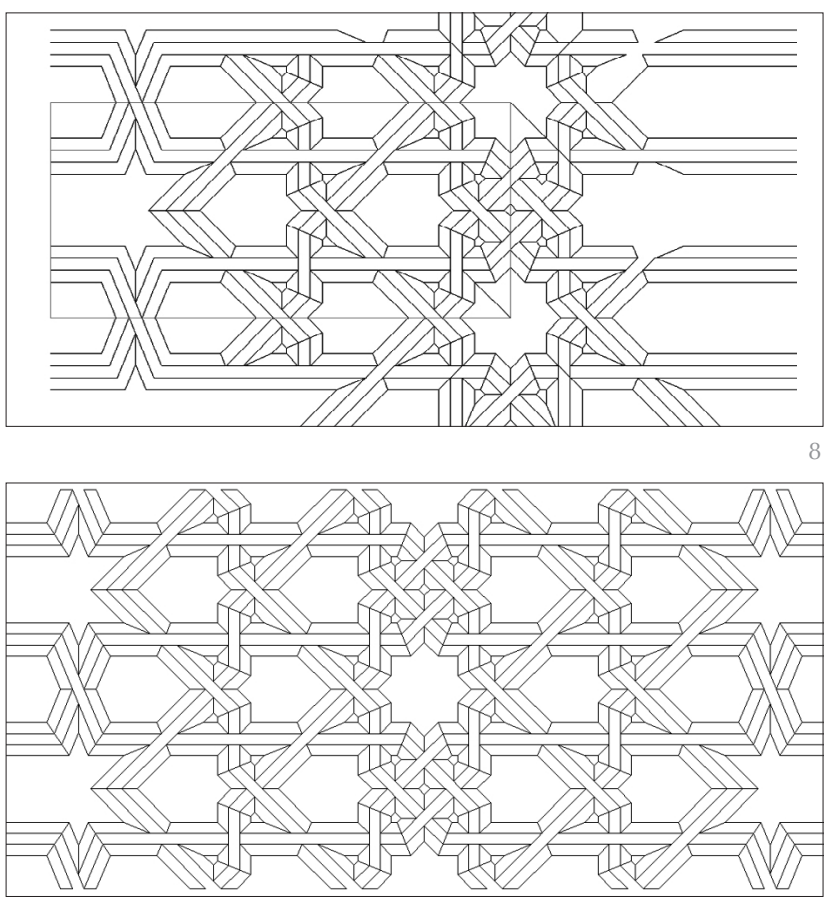
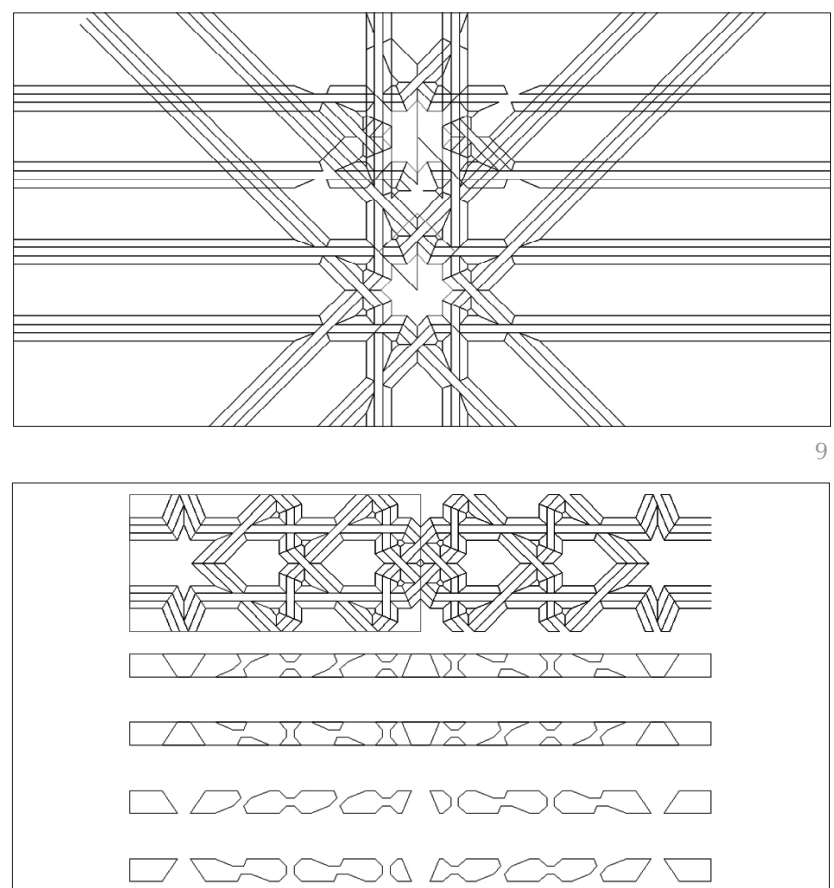

8. En base a las pautas de corte de los nudillos se partió de estrellas de 8 alternadas.

9. El espacio entre las estrellas de 8 se resuelve con un lazo de 4 , y el resto se desculata con un lazo leonés.

10. Con este procedimiento quedaba definida la geometría del trazado y solo restaba confirmarla comprobando los cortes de los nudillos.

11. Se comprueban las secuencias de cortes de los nudillos y se simplifica en un módulo base generador del trazado de lazo para montarlo en el ordenador.

12. El trazado total se ajusta a las medidas reales de la estancia y ya se puede proceder a elaborar todos los planos necesarios para la res tauración completa de la armadura.

10

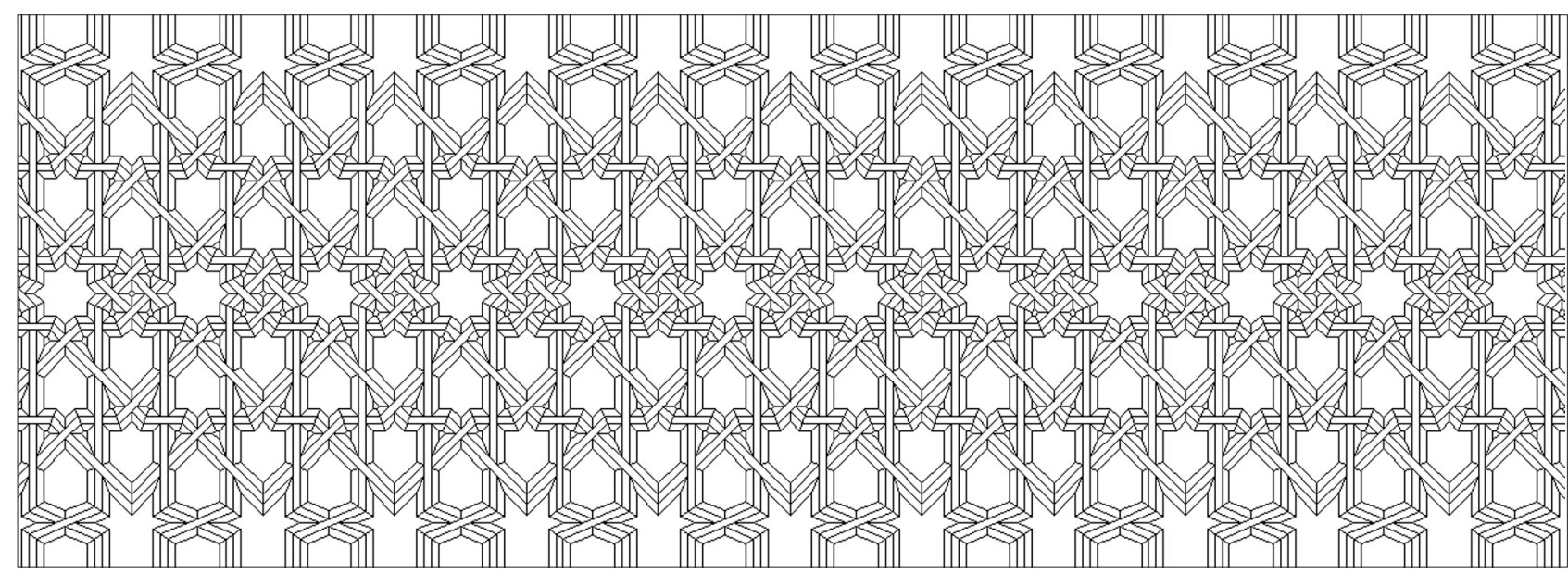



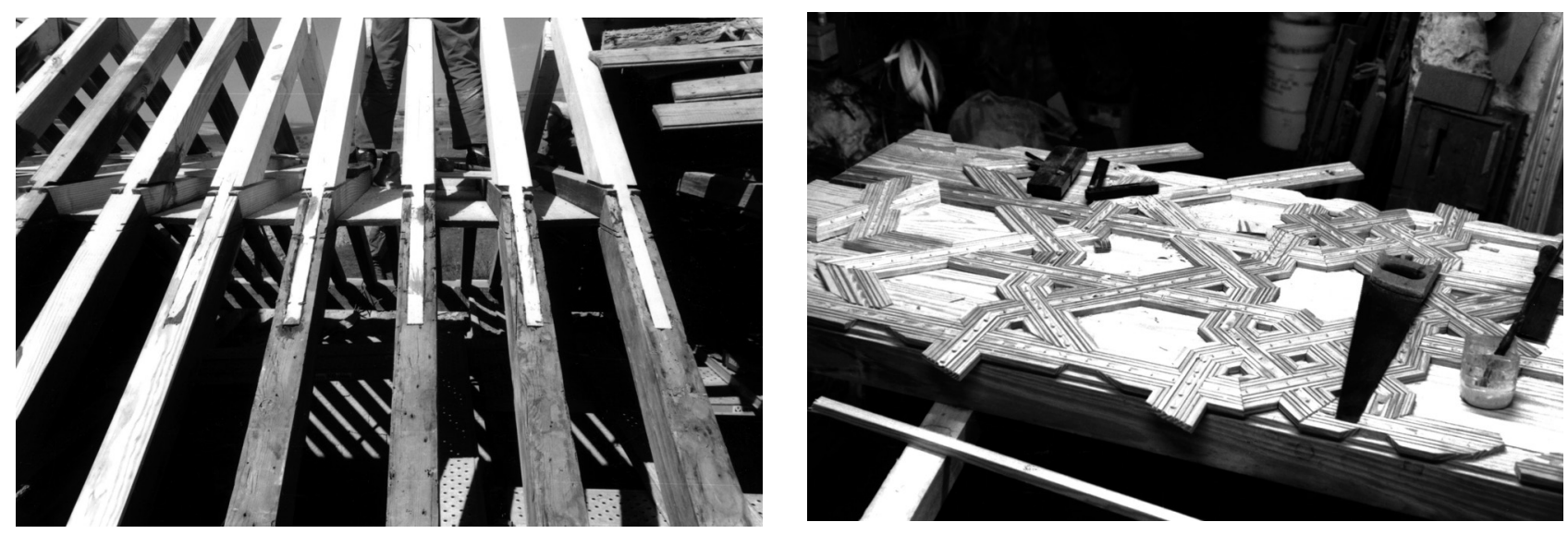

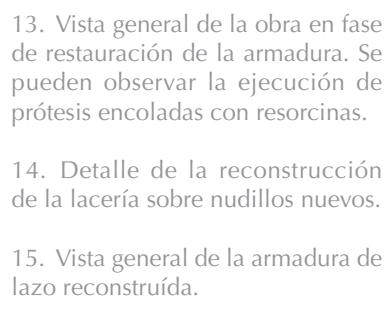

lazo reconstruída.

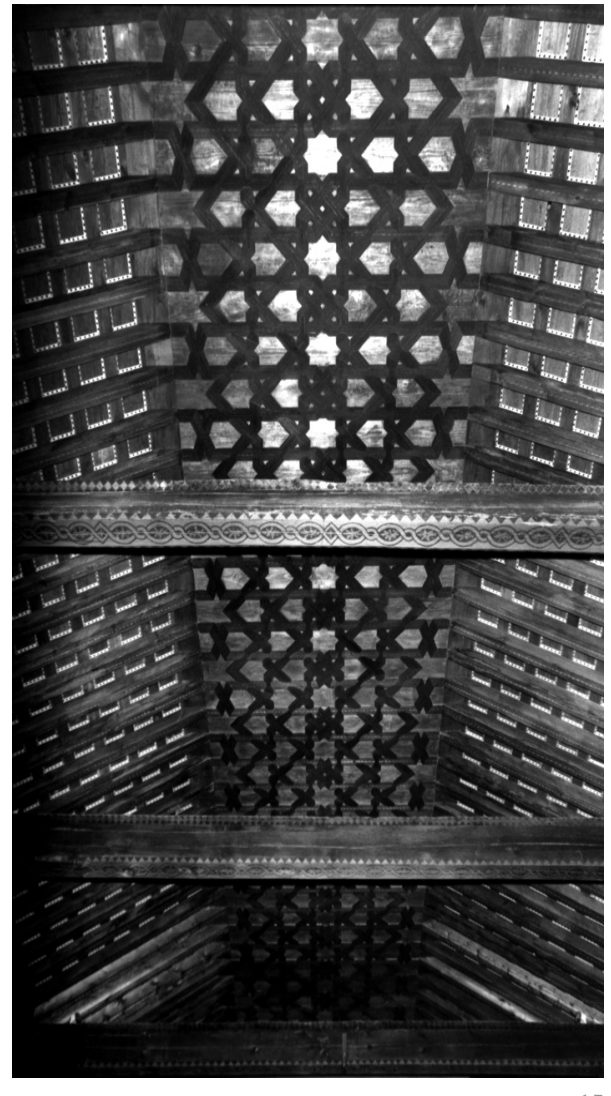

A partir de ahí ya era cuestión de completar todo el paño del almizate o harneruelo, siguiendo las pautas confirmadas y completando todo el conjunto de la lacería del techo que se planteaba reconstruir (Figura 12).

\section{RECONSTRUCCIÓN DE LAARMADURA}

Estos mismos planos fueron los que sirvieron al carpintero para reconstruir el lazo de la armadura, que fue prefabricada totalmente en taller y transportada en paños separados de faldones y almizate, coincidiendo con la modulación de la taravea (lacería) para facilitar su montaje en obra, lo que permitió abaratar enormemente los costes de ejecución.
El comienzo de las obras y el desmantelamiento de la cobertura puso de manifiesto el verdadero estado de conservación del artesonado, muy atacado por insectos xilófagos y por hongos de pudrición, debido a las filtraciones de agua sobre todo los estribos y apoyos de alfardas que estaban ocultos por los aliceres del arrocabe. Con las piezas que pudieron recuperarse de todo el conjunto de la armadura original (Figura 13), se pudo montar un pequeño tramo hacia la cabecera de la iglesia. El resto de la armadura se construyó ex novo, siguiendo el patrón o modelo del existente, cuando en proyecto lo que se preveía era básicamente una restauración y no una reconstrucción. El trabajo se completó con labores decorativas de pigmentación de la madera siguiendo los métodos muy similares a los utilizados en la época de su construcción original (Figura 14).

La policromía del artesonado fue restaurada en aquellas partes originales conservadas, mientras que la madera nueva fue policromada siguiendo técnicas similares a las de la armadura original, si bien un tratamiento en profundidad de este tema se sale fuera del ámbito de este artículo.

\section{CONCLUSIONES}

La posibilidad de recuperar lagunas cuando procedemos a la restauración de edificios históricos puede ser puesta en tela de juicio cuando la certidumbre entre la hipótesis de reconstrucción propuesta y la realidad histórica posible no es suficientemente grande. A medida que aumentan las dudas, aumenta el riesgo de reconstruir algo que nunca existió, con lo que su viabilidad queda en entredicho. Sin embargo en el caso de los trazados de lazo y debido a sus pautas de regularidad geométrica intrínsecas, nos permite utilizar distintos métodos gráficos de génesis y desarrollo para conseguir una restitución no solo virtual sino constructiva, de estos trazados de lazo. En estos casos, la 
reconstrucción de grandes lagunas queda justificada por la certidumbre científica en la recuperación del trazado (Figura 15).

\section{DATOS DE LA OBRA}

Restauración de la iglesia de Almenara de Tormes (Salamanca)

Monumento Declarado: D. 182/93 de 29 de julio. B.O.E. 27 de agosto de 1993

- Promotor: Junta de Castilla y León. Direcc. Gral de Patrimonio y Promoción Cultural
- Arquitecto de proyecto y director de obra: Miguel Carlos Fernández Cabo

- Contratista: REARASA, Restauración de Retablos y Artesonados, S.A.

- Fecha Proyecto Año 1999

- Fecha Obra Año 2000

\section{AGRADECIMIENTOS}

Agradecemos a la empresa REARASA, Restauración de Retablos y Artesonados, S.A., la aportación desinteresada de las fotografías que se han utilizado en este artículo.

\section{BIBLIOGRAFÍA}

(1) Fernández Cabo, M. C.: Armaduras de cubierta. Ámbito Ediciones, Valladolid, 1997.

(2) Angulo Iñiguez, D.: "Estructuras de cubierta islámicas llegadas a América a través de España: las armaduras con lacería morisca"; Actas XXIII, C.I.H.A., Separata, Granada, 3-8 sept, 1973.

(3) Nuere, E.: La carpintería de lo blanco; Lectura dibujada del primer manuscrito de Diego López de Arenas. Ministerio de Cultura, Madrid, 1985.

(4) Fernández Cabo, M. C.: Armaduras de cubierta en la región leonesa: Bases documentales y criterios para el análisis, clasificación y evolución de las tipologías estructurales de las armaduras de cubierta en la región leonesa. Ph.D. Madrid, 1991.

(5) Nuere, E.: Los cartabones como instrumento exclusivo para el trazado de lacerías", Madrider Mttelilulngen 23, Deutsches Archaelologisches Institut, Mainz, 1982.

(6) López de Arenas, D.: Breve compendio de la carpintería de lo blanco y Tratado de alarifes. Luis Estupiñan, Sevilla, 1633.

(7) Nuere, E.: La carpintería de lazo: lectura dibujada del manuscrito de Fray Andrés de San Miguel. Colegio Oficial de Arquitectos de Málaga, Málaga, 1990.

(8) Álvarez de la Braña, R.: "San Marcos de León", B.S.C.E., II, Valladolid, 1905.

(9) Benavides Moro, N.: "El artesonado de La Bañeza", Archivos Leoneses, Año VII, no 13, ene-jul., 1953.

(10) Candelas, A. L.: Análisis constructivo de la carpintería de armar en la provincia de Huelva; su relación con los tratados de carpintería. Ph.D, Sevilla, 1999. 